\title{
In-situ Liquid-cell TEM Studies of High Entropy Nanomaterials
}

Azadeh Amiri and Reza Shahbazian-Yassar

${ }^{1}$ University of Illinois-Chicago, Chicago, Illinois, United States

Entropy-stabilized high entropy oxides (HEOs) are a new class of metal oxides composed of five or more near equimolar metal cations randomly occupying a single cationic sublattice and oxygen in anionic sublattice ${ }^{1}$. Superior structural stability, the high number of surface configurations and vast compositional space for the fine-tuning of favorable properties make them attractive to be explored as new advanced functional materials ${ }^{2}$. Yet many aspects of the formation of disorderly distributed different atoms in a single-crystal structure is not clear and demands further deep investigations.

In this work, the synthesized transition metal and mixed rear-earth/transition metal HEO nanoparticles (HEO-NPs) based on $\mathrm{Mn}, \mathrm{Fe}, \mathrm{Ni}, \mathrm{Co}, \mathrm{Cu}, \mathrm{Zn}, \mathrm{La}$ and $\mathrm{Ce}$ elements with different compositions through co-precipitation method are studied in order to clearly detect and tailor their nanocrystalline phase structure and evolution as well as their electrochemical behavior as electrode materials. Structural and chemical characterizations at the atomic scale are the key route to explore the relationship between nanostructure, composition change and performance in these multi-element material systems.

In this regard, X-ray diffraction (XRD), aberration-corrected scanning transmission electron microscopy (STEM), energy-dispersive X-ray spectroscopy (EDS) and high angle annular dark field imaging (HAADF) characterization techniques are applied to identify the crystal phase structure, chemical composition and distribution of HEO-NPs. The results of sample characterizations, compositional effect, and corresponding electrochemical behaviors will be presented. In addition to ex-situ studies, we utilized liquid-cell TEM to study the in-situ formation of high entropy nanomaterials in liquid solutions. In this aim, the liquid solution of mixed metal precursors is used in the liquid cell holder to monitor their nucleation and growth during the formation of high entropy nanoparticles. This method can considerably level up our understanding of the mechanisms involved during the formation and structural evolution of multielement high entropy materials.
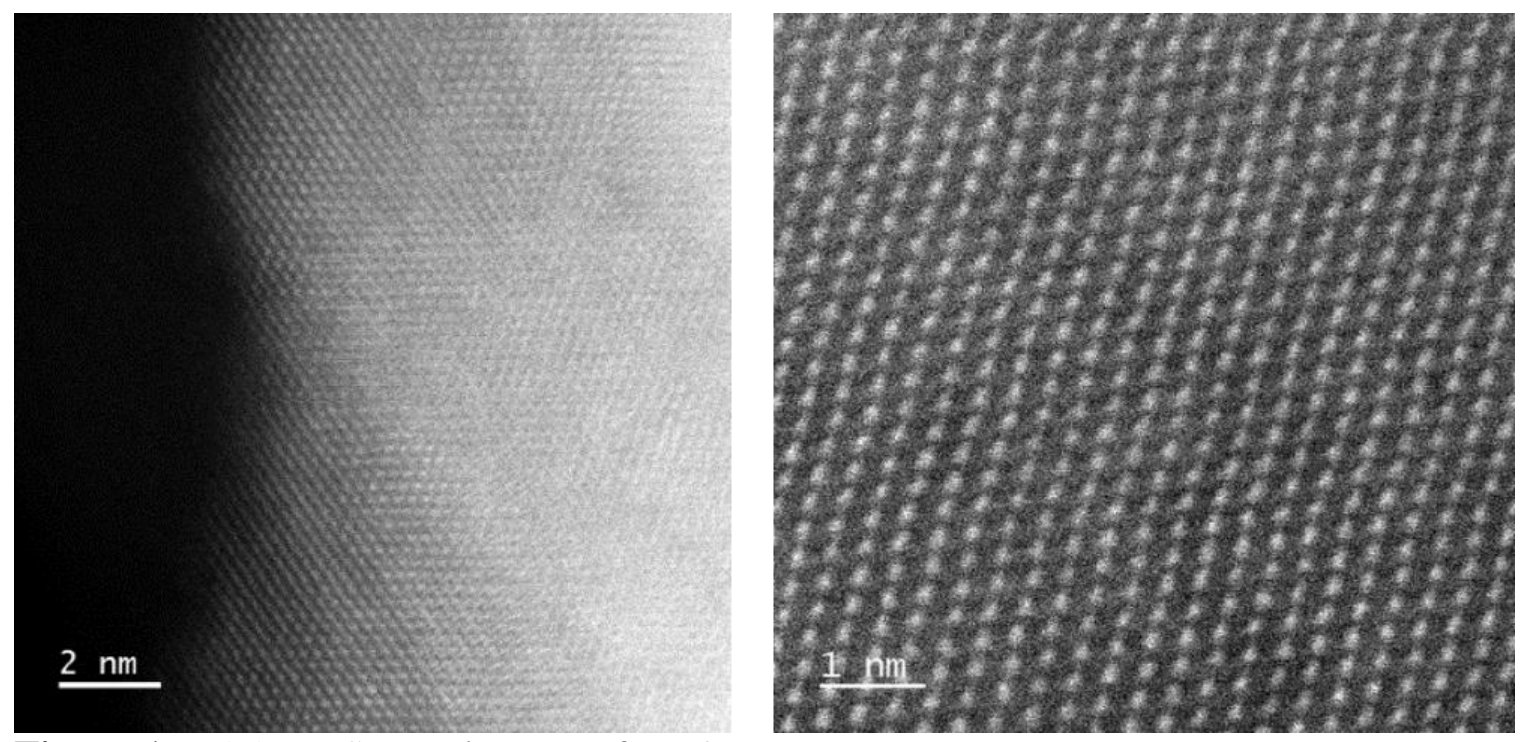

Figure 1. HAADF-STEM images of HEO 

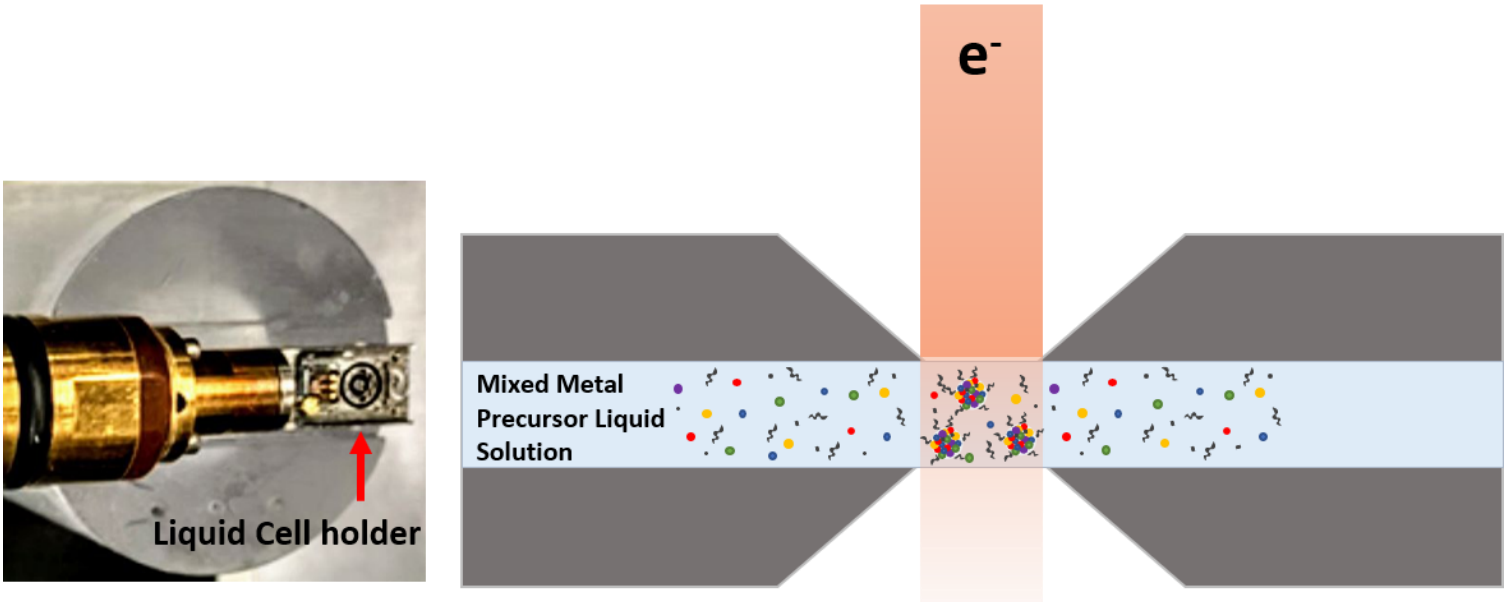

Figure 2. Liquid Cell holder and schematic of in-situ TEM setup for monitoring multi-metallic nanoparticles

\section{References}

(1) Rost, C. M.; Sachet, E.; Borman, T.; Moballegh, A.; Dickey, E. C.; Hou, D.; Jones, J. L.; Curtarolo, S.; Maria, J. Entropy-Stabilized Oxides. Nat. Commun. 2015, 6, 1-8. https://doi.org/10.1038/ncomms9485.

(2) Sarkar, A.; Wang, Q.; Schiele, A.; Chellali, M. R.; Bhattacharya, S. S.; Wang, D.; Brezesinski, T.; Hahn, H.; Velasco, L.; Breitung, B. High-Entropy Oxides : Fundamental Aspects and Electrochemical Properties. 2019, 1806236. https://doi.org/10.1002/adma.201806236. 